\title{
Magnetization relaxation in (Ga,Mn)As ferromagnetic semiconductors
}

\author{
Jairo Sinova, ${ }^{1}$ T. Jungwirth ${ }^{2,3}$ X. Liu,${ }^{4}$ Y. Sasaki,${ }^{4}$ J. K. Furdyna, ${ }^{4,3}$ W. A. Atkinson,,${ }^{5}$ and A. H. MacDonald ${ }^{3}$ \\ ${ }^{1}$ Department of Physics, Texas A\&M University, College Station, Texas 77843-4242, USA \\ ${ }^{2}$ Institute of Physics ASCR, Cukrovarnická 10, 16253 Praha 6, Czech Republic \\ ${ }^{3}$ Department of Physics, University of Texas at Austin, Austin, Texas 78712-0264, USA \\ ${ }^{4}$ Department of Physics, University of Notre Dame, Notre Dame, Indiana 46556, USA \\ ${ }^{5}$ Department of Physics, Trent University, Ontario, Canada K9J $7 B 8$
}

(Received 19 August 2003; revised manuscript received 17 November 2003; published 27 February 2004)

\begin{abstract}
We describe a theory of Mn local-moment magnetization relaxation due to $p$ - $d$ kinetic-exchange coupling with the itinerant-spin subsystem in the ferromagnetic semiconductor (Ga,Mn)As alloy. The theoretical Gilbert damping coefficient implied by this mechanism is calculated as a function of Mn-moment density, hole concentration, and quasiparticle lifetime. Comparison with experimental ferromagnetic resonance data suggests that in annealed strongly metallic samples, $p$ - $d$ coupling contributes significantly to the damping rate of the magnetization precession at low temperatures. By combining the theoretical Gilbert coefficient with the values of the magnetic anisotropy energy, we estimate that the typical critical current for spin-transfer magnetization switching in all-semiconductor trilayer devices can be as low as $\sim 10^{5} \mathrm{~A} \mathrm{~cm}^{-2}$.
\end{abstract}

DOI: 10.1103/PhysRevB.69.085209

PACS number(s): 73.20.Mf, 73.40.-c, 85.75.-d

\section{INTRODUCTION}

The Gilbert coefficient describes the damping of smallangle magnetization precession and is one of the key parameters that characterizes collective magnetization dynamics in a ferromagnet. Early theories of magnetization dynamics in transition metals viewed exchange coupling $(\propto \mathbf{S} \cdot \mathbf{s})$ between local moment $d$-shell spins $\mathbf{S}$ and itinerant $s-p$ band spins $\mathbf{S}$ as a key relaxation mechanism. ${ }^{1}$ We now recognize that this model needs to be elaborated for transition metals to account for the itinerant character of their $d$-electrons. Models of $d$-shell local moments that are exchange coupled to itinerant $s-p$ band electrons have, however, been resurrected recently because they provide a good description of ferromagnetism in many diluted magnetic semiconductors (DMS's), (Ga,Mn)As in particular., ${ }^{2,3}$ Exchange-coupling between local moments and itinerant electrons should also contribute significantly to Gilbert damping in these new ferromagnetic systems. The elementary process for this damping mechanism is one in which a local-moment magnon is annihilated by exchange interaction with a band electron that suffers a spin flip. This process cannot by itself change the total magnetic moment since the exchange Hamiltonian commutes with the total spin $\mathbf{S}+\mathbf{s}$. Net relaxation of the magnetization requires another independent process in which the itinerant electron spin relaxes through spin-orbit interactions.

Recent experiments and $a b$ initio calculations ${ }^{4}$ have established that for doping levels up to several percent, a substitutional Mn impurity in GaAs introduces five strongly localized $d$ electrons and a delocalized hole in the As/Ga $p$ band, and that ferromagnetic coupling between the $S=5 / 2 \mathrm{Mn}$ moments is mediated by the $p-d$ kinetic exchange. Hence, we model $^{5}$ the electronic structure of the free carriers by that of the host material, implicitly assuming a shallow acceptor picture. The free-carrier quasiparticles are $p-d$ exchange coupled to the local moments with strength $^{3} J_{p d}$ $\approx 55 \mathrm{meV} \mathrm{nm}^{3}$, and have a finite life time that can be estimated perturbatively. This theoretical picture leads to an ac- curate description of many thermodynamic and transport properties of optimally annealed $(\mathrm{Ga}, \mathrm{Mn})$ As samples, such as the measured transition temperature ${ }^{6,7}$ the anomalous Hall effect, ${ }^{3,8}$ anisotropic magnetoresistance, ${ }^{8}$ and magnetooptical properties. ${ }^{6,9}$ Particularly important in justifying the present theory are results for the magnetocrystalline anisotropy, ${ }^{10}$ spin stiffness, ${ }^{11,12}$ and Bloch domain width ${ }^{13}$ that all agree well with experiment. These parameters follow from the long-wavelength limit of the theory of the Mn spinwave dispersion, and reflect the retarded and nonlocal character of the valence-band-hole mediated interactions between Mn moments. ${ }^{11}$ The Gilbert damping of magnetization precession discussed here is the aspect of this long-wavelength collective magnetization dynamics that is most directly dependent on valence-band spin-orbit coupling.

In Sec. II of this paper, we present a fully microscopic theory of the kinetic-exchange contribution to the Gilbert coefficient in DMS's. By comparing the linear response predicted by the classical phenomenological Landau-LifshitzGilbert (LLG) equation with microscopic linear-response theory, we identify the Gilbert coefficient with the dissipative part of a susceptibility diagram.

In Sec. III we present experimental ferromagnetic resonance (FMR) data ${ }^{12,14}$ recorded as a function of temperature and external magnetic-field strength and orientation. Since the frequency dependence of the FMR linewidth is not available, ${ }^{12,14}$ we are unable to experimentally decouple inhomogeneous FMR broadening and the intrinsic Gilbert damping contributions to the linewidth to make a quantitative comparison with the theory. Nevertheless, the data indicate that the magnetic inhomogeneity contribution is largely suppressed in the more metallic, annealed samples and that much of the observed low-temperature FMR linewidth in these samples can be explained by damping of the magnetization precession mediated through the $p-d$ coupling.

By adding a spin-torque term ${ }^{15}$ to the LLG equation, we estimate in Sec. IV that the typical critical current for magnetization switching due to spin-transfer torques in an all- 
semiconductor trilayer device consisting of magnetically "soft" and "hard" DMS layers separated by a nonmagnetic spacer will be $\sim 10^{5} \mathrm{~A} \mathrm{~cm}^{-2}$. In metals, this spin-transfer effect is currently the focus of a considerable experimental ${ }^{16}$ and theoretical ${ }^{17}$ research. Spin-transfer switching has not yet been demonstrated in all-semiconductor systems, but the effect promises to have a richer phenomenology in this case because of the flexibility of semiconductor ferromagnet materials, and because of the possibility of combining spintransfer with other semiconductor circuit functionalities. ${ }^{18,19}$ The relatively low critical currents we predict for semiconductors may also circumvent the incomplete magnetization switching encountered in metallic magnetic tunnel junctions that occurs due to the interference of strong self-field effects with the spin-transfer torques. ${ }^{20}$ The paper concludes with a brief summary of our results.

\section{THEORY OF THE GILBERT DAMPING}

Semiclassical LLG linear response. The phenomenological LLG equation for collective magnetization dynamics is

$$
\frac{d \mathbf{M}}{d t}=-\frac{g \mu_{B}}{\hbar} \mathbf{M} \times \mathbf{B}_{e f f}+\frac{\alpha}{M} \mathbf{M} \times \frac{d \mathbf{M}}{d t},
$$

where $\mathbf{M}$ is the local Mn-moment magnetization, $\mathbf{B}_{\text {eff }}=$ $-\partial E / \partial \mathbf{M}$ is the effective magnetic field, $g$ is the Landé $g$-factor, $\mu_{B}$ is the Bohr magneton, and $\alpha$ is the phenomenological Gilbert damping coefficient. Unless $\alpha$ depends strongly on the orientation of the magnetization ${ }^{21}$ or if the magnetization is not fully aligned with the external static magnetic field, the Gilbert damping rate, observed in experiment through a frequency-dependent FMR linewidth, is independent of the static field and of the details of magnetic anisotropies present in the sample. ${ }^{22}$ This allows us to assume in this section a simple geometry in which the anisotropy fields are represented by a single, uniaxial anisotropy energy density coefficient $U$. For small fluctuations of the Mn magnetization orientation around the easy axis, Eq. (1) can be used to derive a phenomenological response function of the magnetic system to a weak transverse field. For zero external static magnetic field the corresponding inverse susceptibility reads

$$
\chi^{-1}=\frac{\hbar}{\left(g \mu_{B}\right)^{2} N_{M n} S}\left(\begin{array}{cc}
\widetilde{U}-i \alpha \omega & -i \omega \\
i \omega & \widetilde{U}-i \alpha \omega
\end{array}\right),
$$

where $\widetilde{U}=U /\left(\hbar N_{M n} S\right), N_{M n}=4 x / a_{l c}^{3}$ is the density of uniformly distributed $\mathrm{Mn}$ moments in $\mathrm{Ga}_{1-x} \mathrm{Mn}_{x} \mathrm{As}\left(a_{l c}\right.$ is the GaAs lattice constant), and $\omega$ is the frequency of the external rf field perturbation.

Microscopic theory. We derive the zero-temperature quantum response function from our effective Hamiltonian theory and obtain a microscopic expression for $\alpha$ by equating the quantum-mechanical response function to the classical one in the uniform $\omega \rightarrow 0$ limit. We start by writing a quantum ana$\log$ of Eq. (1) using the linear-response theory,

$$
\begin{aligned}
\left\langle M_{x}(\mathbf{r}, t)\right\rangle= & -\frac{i}{\hbar} \int_{-\infty}^{\infty} d t^{\prime} \int d \mathbf{r}^{\prime}\left(\left\langle\left[M_{x}(\mathbf{r}, t),\right.\right.\right. \\
& \left.\left.-M_{x}\left(\mathbf{r}^{\prime}, t^{\prime}\right) B_{x}\left(t^{\prime}\right)\right]\right\rangle+\left\langle\left[ M_{x}(\mathbf{r}, t),\right.\right. \\
& \left.\left.\left.-M_{y}\left(\mathbf{r}^{\prime}, t^{\prime}\right) B_{y}\left(t^{\prime}\right)\right]\right\rangle\right) \theta\left(t-t^{\prime}\right),
\end{aligned}
$$

which leads to the retarded transverse susceptibility:

$$
\chi_{i, j}^{R}\left(\mathbf{r}, t \mid \mathbf{r}^{\prime}, t^{\prime}\right)=\left(g \mu_{B}\right)^{2} \frac{i}{\hbar}\left\langle\left[S_{i}(\mathbf{r}, t), S_{j}\left(\mathbf{r}^{\prime}, t^{\prime}\right)\right]\right\rangle \theta\left(t-t^{\prime}\right) .
$$

Here $i=x, y$ and $S_{i}(\mathbf{r}, t)=M_{i}(\mathbf{r}, t) /\left(g \mu_{B}\right)$ are the Mn transverse spin-density operators.

To evaluate the correlation function (4) we use the Holstein-Primakoff boson representation of the spin operators assuming small fluctuations around the mean-field ordered state, $S^{+}=b \sqrt{2 N_{\mathrm{Mn}} S}$ and $S^{-}=b^{\dagger} \sqrt{2 N_{\mathrm{Mn}} S}\left[S_{x}=\left(S^{+}\right.\right.$ $\left.\left.+S^{-}\right) / 2, S_{y}=\left(S^{+}-S^{-}\right) / 2 i\right]$, and choosing the $\hat{z}$ direction as the quantization axis. After integrating out the itinerant carrier degrees of freedom within the coherent-state pathintegral formalism of the many-body problem we obtain the partition function, $Z=\int \mathcal{D}[\bar{z} z] \exp (-S[\overline{z z}])$, with the action given to quadratic order in $z$ and $\bar{z}$ (the complex numbers representing the bosonic degrees of freedom) by

$$
S[\bar{z} z]=1 / \beta V \sum_{m, \mathbf{k}} \bar{z}\left(\mathbf{k}, \Omega_{m}\right)\left(-i \Omega_{m}+\Pi\left(i \Omega_{m}\right)\right) z\left(\mathbf{k}, \Omega_{m}\right) .
$$

In Eq. (5), the first term is the standard Berry's phase and the second term is the itinerant carrier spin-polarization diagram for the high-symmetry case where the cross terms of the form $\overline{z z}$ and $z z$ vanish in $S[\bar{z} z]:^{11}$

$$
\begin{aligned}
\Pi(i \Omega)= & \frac{N_{M n} J_{p d}^{2} S}{2 \beta} \int \frac{d^{3} k}{(2 \pi)^{3}} \sum_{m, a, b} \mathcal{G}_{a}\left(i \omega_{m}, \mathbf{k}\right) \mathcal{G}_{b}\left(i \omega_{m}\right. \\
& +i \Omega, \mathbf{k})\left|\left\langle\phi_{a}(\mathbf{k})\left|s^{+}\right| \phi_{b}(\mathbf{k})\right\rangle\right|^{2} .
\end{aligned}
$$

Here $\mathcal{G}_{a}(z, \mathbf{k})$ is the single-particle band Green's function and $\phi_{a}(\mathbf{k})$ are the band eigenstates. From the partition function we compute directly the imaginary time response functions at finite Matsubara frequencies which after their corresponding analytic continuations yield

$$
\begin{gathered}
\chi_{x x}^{R}(\omega)=-\left(g \mu_{B}\right)^{2} \frac{N_{M n} S}{2 \hbar} \frac{2 \Pi^{\mathrm{ret}}(\omega)}{\omega^{2}+i \delta-\Pi^{\mathrm{ret} 2}(\omega)}, \\
\chi_{x y}^{R}(\omega)=-i\left(g \mu_{B}\right)^{2} \frac{N_{M n} S}{2 \hbar} \frac{2 \omega}{\omega^{2}+i \delta-\Pi^{\mathrm{ret} 2}(\omega)} .
\end{gathered}
$$

Here $\Pi^{\text {ret }}$ (calculated below) describes mathematically the retarded interaction between the Mn bosonic degrees of freedom due to the $p$ - $d$ kinetic exchange with valence-band holes.

Connecting classical phenomenology and microscopic theory. Inverting the retarded susceptibility in Eq. (7) for the uniform $(\mathbf{k}=\mathbf{0})$ precession mode, we obtain 


$$
\begin{gathered}
\chi_{x x}^{R-1}(\omega)=\frac{\hbar \Pi^{\mathrm{ret}}(\omega)}{\left(g \mu_{B}\right)^{2} N_{M n} S}, \\
\chi_{x y}^{R-1}(\omega)=-i \frac{\hbar \omega}{\left(g \mu_{B}\right)^{2} N_{M n} S} .
\end{gathered}
$$

Comparing Eqs. (8) and (2), we obtain the microscopic contribution to the Gilbert coefficient from kinetic-exchange coupling:

$$
\alpha=-\lim _{\omega \rightarrow 0}\left[\operatorname{Im} \Pi^{\mathrm{ret}}(\omega) / \omega\right] .
$$

Note that $\chi_{x y}^{R-1}$ is explicitly equal to the off-diagonal element of $\chi^{-1}$ in Eq. (2) and that, also consistently, the real part of $\Pi^{\text {ret }}(\omega)$, in the limit of $\omega \rightarrow 0$, gives the magnetocrystalline contribution to the anisotropy energy $\widetilde{U}$, as explained in detail in Ref. 11.

To compute $\Pi^{\text {ret }}(\omega)$, we take into account the effects of disorder present in the system perturbatively by accounting for the finite lifetime of band quasiparticles, which for simplicity we characterize by a single number $\Gamma$. The quasiparticle broadening $\Gamma$ was chosen to be in the range estimated in previous detailed studies of transport properties of these systems, which achieve good agreement with experiment. ${ }^{8}$ The single-particle Green's function for the valence-band quasiparticles is thus written as $\mathcal{G}_{a}(\mathbf{k}, z)=\int_{-\infty}^{\infty} d \omega^{\prime} /$ $(2 \pi) A_{a}\left(\omega^{\prime}, \mathbf{k}\right) /\left(z-\omega^{\prime}\right)$ with a spectral function $A_{a}(\epsilon, \mathbf{k})$ $=\Gamma /\left[\left(\epsilon-\epsilon_{a, \mathbf{k}}\right)^{2}+\Gamma^{2} / 4\right]$. In the present case we take the valence-band electronic structure to be described by the sixband Kohn-Luttinger Hamiltonian in the presence of an effective kinetic-exchange field $h_{e f f}=J_{p d} N_{M n}\langle S\rangle .{ }^{10}$ In a collinear ferromagnetic state and zero-temperature $\langle S\rangle=S$, and we obtain

$$
\begin{aligned}
\alpha= & \lim _{\omega \rightarrow 0} \frac{N_{M n} J_{p d}^{2} S}{4 \hbar \omega} \int \frac{d^{3} k}{(2 \pi)^{3}} \sum_{a, b}\left|\left\langle\phi_{a}(\mathbf{k})\left|s^{+}\right| \phi_{b}(\mathbf{k})\right\rangle\right|^{2} \\
& \times \int \frac{d \boldsymbol{\epsilon}}{2 \pi} A_{a, \mathbf{k}}(\epsilon) A_{b, \mathbf{k}}(\epsilon+\hbar \omega)[f(\boldsymbol{\epsilon})-f(\epsilon+\hbar \omega)] \\
= & \frac{J_{p d} h_{e f f}}{4 \hbar} \int \frac{d^{3} k}{(2 \pi)^{3}} \sum_{a, b}\left|\left\langle\phi_{a}(\mathbf{k})\left|s^{+}\right| \phi_{b}(\mathbf{k})\right\rangle\right|^{2} \\
& \times A_{a, \mathbf{k}}\left(\boldsymbol{\epsilon}_{F}\right) A_{b, \mathbf{k}}\left(\boldsymbol{\epsilon}_{F}\right) .
\end{aligned}
$$

In choosing a spin- and band-independent lifetime, we are implicitly appealing to the dominance of spin-independent Coulomb scattering off $\mathrm{Mn}$ acceptors and interstitials as the dominant ${ }^{8}$ scattering mechanism. Spin-orbit interactions enter through their presence in the intrinsic bands rather than through spin-flip quasiparticle scattering events. In this model, the Gilbert coefficient in Eq. (10) has intraband and interband contributions that have qualitatively different disorder dependences, as illustrated in Fig. 1. Note that the terminology we use here recognizes that no band has spin character sufficiently definite to justify the usual distinction between majority and minority spin bands. The intraband

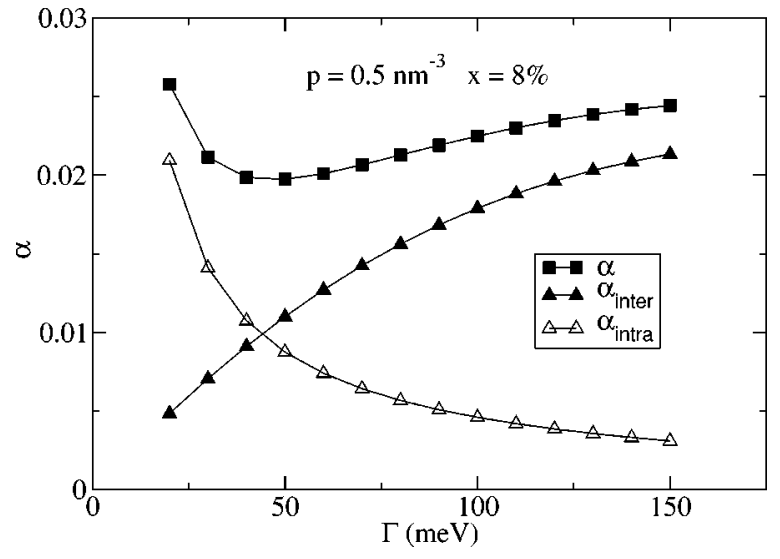

FIG. 1. Total Gilbert damping coefficient $\alpha$, interband contribution $\alpha_{\text {inter }}$, and intraband contribution $\alpha_{\text {intra }}$ as a function of quasiparticle lifetime broadening $\Gamma$ for a carrier density of $0.5 \mathrm{~nm}^{-3}$ and $x=8 \%$.

term we refer to here would be spin-flip scattering within a given spin-split band in the more usual language and is present only because of intrinsic spin-orbit coupling in the host semiconductor bands. The intraband contribution to $\alpha$ is proportional to $1 / \Gamma$ at small $\Gamma$, i.e., proportional to the conductivity rather than the resistivity, and would dominate the damping if disorder were weak. The interband contribution to $\alpha$, on the other hand, requires disorder to breach the wave-vector separation between different bands at the Fermi energy and is an increasing function of $\Gamma$. The overall dependence of the Gilbert coefficient on the sample's mobility is non-monotonic, as illustrated in Fig. 1, with the position of the minimum depending on both hole and Mn-moment densities, and on other parameters of the DMS system.

The complexity and tunability of the Gilbert coefficient in DMS's is illustrated in Fig. 2 where we plot $\alpha$ as a function of the hole density for $\Gamma=150$ and $50 \mathrm{meV}$ and for $\mathrm{Mn}$ doping $x=2-8 \%$. These parameter values bracket the range typical for metallic (Ga,Mn)As DMS's. The theory curves in Fig. 2 predict that $\alpha$ increases with increasing hole density because of the higher quasiparticle density of states at larger densities. The dependence of the Gilbert coefficient on $x$ is more complex. The prefactor $h_{\text {eff }}$ in Eq. (10) reflects the proportionality of the kinetic-exchange coupling to the Mn spin density and causes the Gilbert damping implied by this mechanism to decrease with decreasing $x$ at low Mn doping. This behavior is clearly seen in Fig. 2(a) for $x \leqslant 6 \%$. On the other hand, an opposite trend is predicted for higher Mnmoment densities where the effect of $h_{\text {eff }}$ on the intraband and interband matrix elements in Eq. (10) takes over. In that case, larger $h_{\text {eff }}$ values lead to a reduced spin mixing in the quasiparticle bands and, therefore, to smaller Gilbert damping rates. This implicit dependence of $\alpha$ on $h_{\text {eff }}$ is more dramatic in higher-quality samples.

We expect that the above kinetic-exchange mechanism of the Gilbert damping will dominate at low temperatures where other mechanisms such as magnon-magnon interactions vanish. At temperatures close to the Curie temperature, on the other hand, the contribution to magnetization precession damping due to the kinetic-exchange coupling can be 

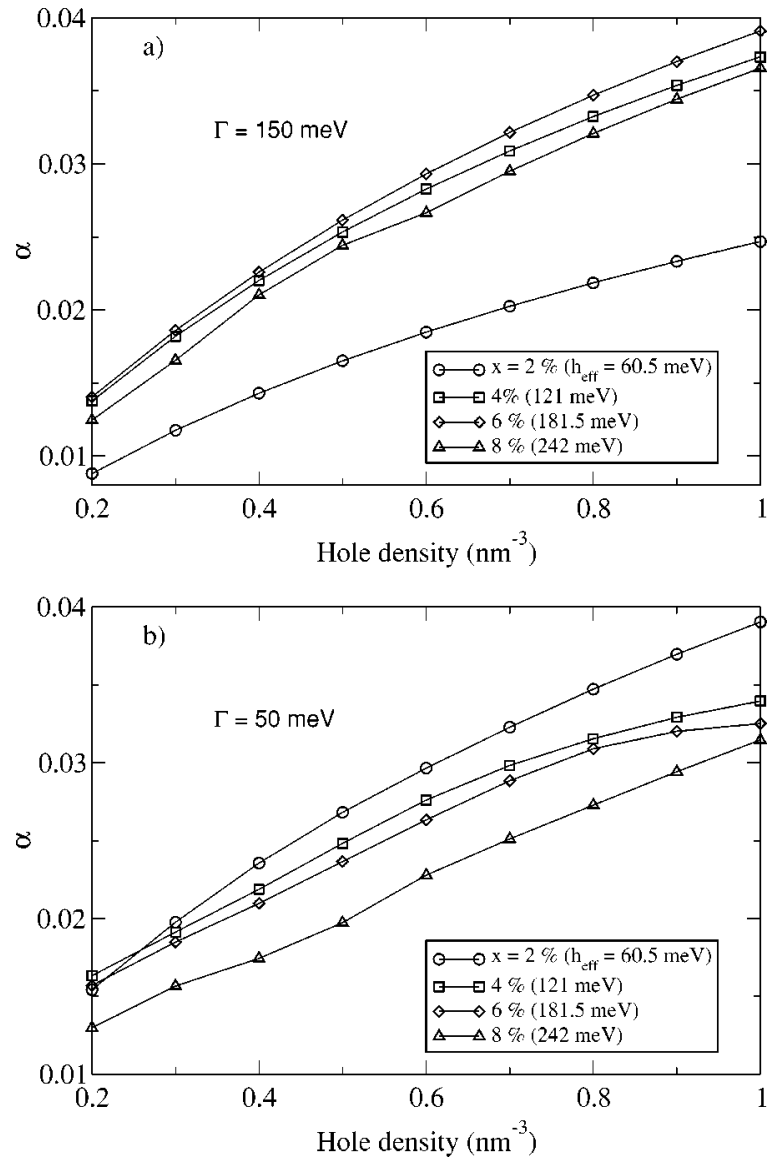

FIG. 2. Gilbert damping coefficient $\alpha$ as a function of carrier density for $x=2-8 \%$ and for quasiparticle lifetime broadening of $150 \mathrm{meV}$ (a) and $50 \mathrm{meV}$ (b).

ignored. The argument is based on an approximation that combines our zero-temperature microscopic theory of $\alpha$ with a finite-temperature mean-field description of $h_{\text {eff }} \cdot{ }^{10}$ Within the mean-field model, $h_{\text {eff }}$ is proportional to mean Mn spinpolarization $\langle S\rangle$ whose temperature dependence is given by the Brillouin function with a temperature-dependent mean field. ${ }^{10}$ The curves in Fig. 2 can therefore be approximately assigned also to a $(\mathrm{Ga}, \mathrm{Mn}) \mathrm{As}$ DMS system where the effective-field value changes through the temperaturedependent average $\mathrm{Mn}$-spin polarization rather than through the Mn-doping parameter $x$. Large values of $h_{\text {eff }}$ correspond to low temperatures in this picture and, as seen from Fig. 2, the temperature dependence of $\alpha$ in this regime is quite complex and sensitive to details of the DMS sample structure. Generally, Fig. 2 suggests an initial increase of $\alpha$ with increasing temperature in samples with a large density of $\mathrm{Mn}$ moments, a nearly constant $\alpha$ for intermediate doping, and a suppression of $\alpha$ in samples with low Mn content. At high temperatures (small $h_{e f f}$ ), the kinetic-exchange-driven Gilbert damping rate will gradually decrease towards zero with increasing temperature.

\section{EXPERIMENTAL FMR LINEWIDTH}

We now discuss our experimental FMR linewidth data recorded for the $120 \mathrm{~nm}$ thin $\mathrm{Ga}_{1-x} \mathrm{Mn}_{x}$ As layer with $x$

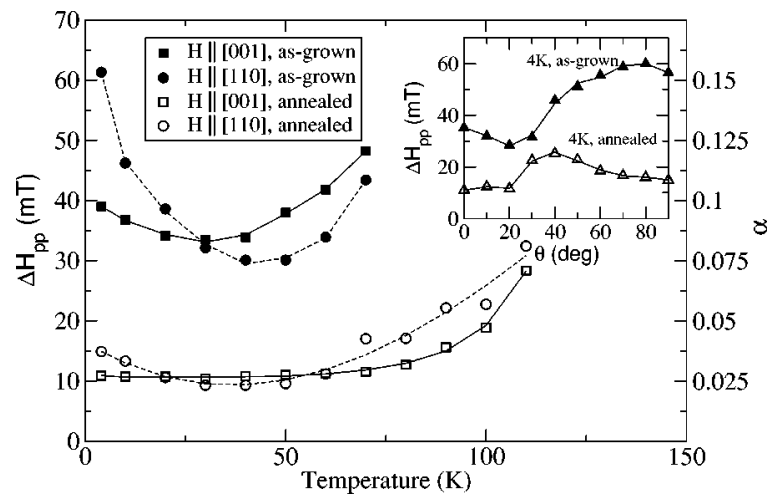

FIG. 3. Experimental peak-to-peak FMR linewidth in as-grown (filled symbols) and annealed (open symbols) $\mathrm{Ga}_{0.92} \mathrm{Mn}_{0.08} \mathrm{As}$ samples measured as a function of temperature for [001] and [110] dc magnetic-field orientations (main plot) and as a function of the field angle at $4 \mathrm{~K}$ (inset).

$=8 \%$ grown on GaAs (001) substrate. The FMR measurements were carried out at $9.46 \mathrm{GHz}$, with the external dc magnetic field applied at different angles $\theta$ with respect to the growth axis $(\theta=0$ corresponds to the [001] crystal direction and $\theta=90^{\circ}$ to the [110] direction). The Mn concentration in the sample was estimated from x-ray-diffraction measurement of the lattice constant. The critical temperature in the as-grown $\left(T_{c}=65 \mathrm{~K}\right)$ and annealed $\left(T_{c}=110 \mathrm{~K}\right)$ samples were determined from superconducting quantum interference device magnetization measurements. A more detailed description of the sample properties and of our experimental setup can be found elsewhere. ${ }^{14}$ To analyze the measured peak-to-peak FMR linewidth $\Delta H_{p p}$, plotted in Fig. 3, we recall the following general relation between $\Delta H_{p p}$ and $\alpha:^{22,23}$

$$
\Delta H_{p p}(\omega)=\Delta H_{p p}(0)+\frac{2}{\sqrt{3}} \frac{\omega}{g \mu_{B}} \alpha .
$$

Here $\Delta H_{p p}(0)$ describes broadening due to sample inhomogeneity which is assumed to be frequency independent $t^{23,24}$ but can depend on the dc field orientation. The second term in Eq. (11) arises from the Gilbert damping term in the LLG equation (1), which gives a contribution to the FMR linewidth which is linearly proportional to $\omega$ and independent of the static magnetic-field direction, if the magnetization is aligned with the field ${ }^{24}$ (and the dependence of $\alpha$ on the magnetization orientation can be neglected, as mentioned in the preceding section ${ }^{21}$ ). This condition is satisfied in our sample since the FMR resonance field is larger than the magnetic field at which saturation magnetizations for different field orientations coincide. ${ }^{14}$

The strong dependence of the FMR linewidth on the field orientation in the as-grown sample suggests that magnetic inhomogeneities in the ferromagnetic layer contribute significantly to the FMR broadening. As seen in both the main panel and the inset of Fig. 3, the angle dependence of $\Delta H_{p p}$ becomes weaker in the annealed sample and also the overall magnitude of $\Delta H_{p p}$ is conspicuously reduced. This observation is consistent with the improved quality of the sample (as 
indicated, e.g., by the enhanced $T_{c}$ ) and implies that the leading contribution to the FMR linewidth might in this case come from the homogeneous (Gilbert damping) broadening. The right $y$ axis in the main plot of Fig. 3 represents the experimental Gilbert coefficient obtained from the measured $\Delta H_{p p}$ and from Eq. (11), assuming $\Delta H_{p p}(0)=0$. Experimental low-temperature values of $\alpha$ in the annealed sample are around 0.03. As seen from Fig. 2, these values of the Gilbert coefficient can be fully explained by the $p-d$ kineticexchange mechanism of the damping of magnetization precession. However, because the density of $\mathrm{Mn}$ ions and their distribution in the lattice as well as the density of holes are not precisely known in our sample, a fully quantitative comparison between theory and experiment is not possible. The results suggest that experimental studies of the frequencydependent FMR linewidth in high-quality DMS samples would be very valuable for understanding the complex behavior of the Gilbert damping coefficient predicted in the theoretical part of this paper, and in separating those effects that are arising from the inhomogeneity within the epitaxially grown thin films.

\section{CURRENT-INDUCED MAGNETIZATION SWITCHING}

Using theoretical values for the Gilbert coefficient and anisotropy energy, ${ }^{10}$ we now estimate the critical current for the spin-transfer induced magnetization switching in a ferromagnetic semiconductor multilayer structure. In general, spin-polarized perpendicular-to-plane currents in magnetic multilayer systems can transfer spin between magnetic layers and exert current-dependent torques. For a trilayer structure, arguments based on the conservation of the angular momentum suggest the following form of the torques on the two magnetic layers: ${ }^{15}$

$$
\tau_{1(2)} \propto \frac{I_{s}}{e M V} \hat{\mathbf{M}}_{1(2)} \times\left(\hat{\mathbf{M}}_{1} \times \hat{\mathbf{M}}_{2}\right),
$$

where $\hat{\mathbf{M}}_{1(2)}=\mathbf{M}_{1(2)} / M$ and $I_{s}$ is the spin-polarized electric current. The sign of the torque depends on the sign of the current, so that magnetization vectors in the two magnetic layers can be aligned parallel or antiparallel by current flowing in one or the opposite direction. In a spin-valve structure with one hard and one soft magnetic layer, switching occurs when the torque in the soft layer overcomes the damping and the anisotropy terms.

There have been a series of theoretical papers ${ }^{17}$ aimed at the quantitative description of spin currents and their effects on magnetization switching in metallic spin-valve structures. The theories are based on a two-channel model (spin up and spin down) and account for spin accumulation effects in the magnetic multilayers and spin-transfer effects due to reflection at the ferromagnet/normal layer interface and due to the averaging mechanism associated with rapid precession of electron spins after entering the ferromagnet. The twochannel model is not applicable for semiconductor valence bands with strong spin-orbit coupling, complicating the quantitative description of spin currents in DMS's. Strong spin-orbit coupling leads to a reduced spin-coherence time.
However, the exchange coupling between the Mn moments and hole carriers will make this time larger in ferromagnetic than in nonmagnetic $p$-type semiconductors. Experimentally, magnetic information can be transported by charge carriers in DMS multilayers, despite strong spin-orbit coupling, as demonstrated, e.g., by the observation of the giant magnetoresistance effects. ${ }^{25}$

For an order-of-magnitude estimate of the switching current in a $(\mathrm{Ga}, \mathrm{Mn})$ As-based magnetic trilayer structure, we approximate the spin current as $I_{s} \approx I\langle s\rangle$, where $I$ is the electric current and $\langle s\rangle$ is the mean-field spin polarization of the itinerant holes in the $(\mathrm{Ga}, \mathrm{Mn})$ As layers. ${ }^{10}$ Adding the torque term (12) to Eq. (1) for the soft magnetic layer we obtain an effective damping rate $D=(\widetilde{U} \alpha-\widetilde{I}) /\left(1+\alpha^{2}\right)$, where $\widetilde{I}$ $=I\langle s\rangle /\left(e N_{M n} S V\right)$. An instability occurs at $D=0$ and the corresponding critical current density for the magnetization switching is then given by $j_{c}=e U \alpha d / \hbar\langle s\rangle$. Assuming a thickness $d \sim 10 \mathrm{~nm}$ for the soft ferromagnetic layer and typical parameters of a $\mathrm{Ga}_{0.95} \mathrm{Mn}_{0.05} \mathrm{As}$ DMS, $U$ $\sim 1 \mathrm{~kJ} \mathrm{~m}^{-3}, \alpha \approx 0.02$, and $\langle s\rangle \approx 0.3$, the critical current $j_{c}$ $\sim 10^{5} \mathrm{~A} \mathrm{~cm}^{-2}$. This estimate is two orders of magnitude smaller than critical switching currents characteristic of metallic spin-valves, ${ }^{16,17}$ primarily due to smaller saturation moments and anisotropy energies in the DMS's. Since the resistivities are only $2-3$ orders of magnitude larger in DMS's than in metals, observation of this effect should be experimentally feasible in a ferromagnet/normal/ferromagnet semiconductor spin-valve structure. ${ }^{26}$

We note that achieving low critical currents is particularly important for magnetic tunnel junctions that are used in nonvolatile memory devices. To avoid self-field effects, which lead to a spin vortex state rather than to a complete reversal and therefore to a smaller giant magnetoresistance effect, ${ }^{20}$ the in-plane diameter $r$ of metallic spin-valve devices with critical current densities $j \sim 10^{7} \mathrm{~A} \mathrm{~cm}^{-2}$ must be of the order of $\sim 100 \mathrm{~nm}$. ${ }^{16}$ Magnetic tunnel junctions have resistances too high for applications when patterned to such small sizes. Since the Oersted field scales as $\sim r j$ and the critical currents for spin-torque-induced switching we predict are two orders of magnitude smaller in DMS's than in metals, $1-10 \mu \mathrm{m}$ size semiconductor tunnel junctions might still show sufficiently weak self-field effects and, therefore, a complete current-induced switching.

\section{SUMMARY}

In this paper we have studied magnetization precession damping in ferromagnetic semiconductor $(\mathrm{Ga}, \mathrm{Mn})$ As alloys. We have attempted to employ theoretical analysis combined with existing experimental information to obtain the Gilbert damping coefficient and to predict the scale of critical currents for spin-transfer magnetization-reversal in these systems. In spin-transfer induced reversal, damping of magnetization precession competes with current-induced spin torques and determines the scale of the current required to achieve reversal.

Our theoretical analysis examines the mechanism that we expect to dominate at low temperatures in high-quality samples, due to the coupling of the $d$-level local moments to 
valence-band holes. We derive an explicit expression for the Gilbert coefficient conventionally used to characterize damping in experimental studies, by comparing microscopic linear-response theory with the linear-response limit of the phenomenological Landau-Lifshitz-Gilbert equations, and study how the values predicted by this model for the Gilbert damping coefficient depend on the hole density and on the size of the mean-field exchange interaction experienced by valence-band holes in the ferromagnetic state. We find that the magnitude predicted for the Gilbert coefficient, $\sim 0.03$, is consistent with experiment, but that the observed dependence on the external field and magnetization orientation is larger than can be accounted for by this mechanism. The experimental FMR linewidth appears to have an inhomogeneous broadening contribution that is not included in our theoretical modeling developed for homogeneous bulk systems. The uncertainty that presently exists in the relative importance of these two broadening mechanisms could be reduced by frequency-dependent FMR studies.

In our view, the portion of the FMR linewidth broadening that is due to inhomogeneity should not, to a first approximation, be included in assessing the competition between spin torques and spin-precession damping. We have therefore used our theoretical value for damping in a homogeneous system to estimate the critical currents required for achieving magnetization reversal and obtained $j_{c} \sim 10^{5} \mathrm{~A} \mathrm{~cm}^{-2}$.

\section{ACKNOWLEDGMENTS}

The authors acknowledge helpful discussions with $\mathrm{T}$. Dietl, Z. Frait, and H. Ohno. This work was supported in part by the Welch Foundation, the DOE under Grant No. DEFG03-02ER45958, the Grant Agency of the Czech Republic under Grant No. 202/02/0912, the Research Corporation under Grant No. CC5543, the DARPA SpinS Program, and by the NSF-NIRT under Grant No. DMR-0210519.
${ }^{1}$ C. Kittel and A.H. Mitchell, Phys. Rev. 101, 1611 (1956); A.H. Mitchell, ibid. 105, 1439 (1957).

${ }^{2}$ T. Dietl, Handbook on Semiconductors (Elsevier, Amsterdam, 1994).

${ }^{3}$ H. Ohno, J. Magn. Magn. Mater. 200, 110 (1999).

${ }^{4}$ T.C. Schulthess, Bull. Am. Phys. Soc. K30, 1 (2003); P.H. Dederichs, K. Sato, H. Katayama-Yoshida, and J. Kudrnovský, ibid. S24, 5 (2003).

${ }^{5}$ J. König, J. Schliemann, T. Jungwirth, and A.H. MacDonald, in Electronic Structure and Magnetism of Complex Materials, edited by D.J. Singh and D.A. Papaconstantopoulos (SpringerVerlag, Berlin, 2003).

${ }^{6}$ T. Dietl, H. Ohno, F. Matsukura, J. Cibert, and D. Ferrand, Science 287, 1019 (2000).

${ }^{7}$ T. Jungwirth, J. König, J. Sinova, J. Kučera, and A.H. MacDonald, Phys. Rev. B 66, 012402 (2002); K.W. Edmonds, K.Y. Wang, R.P. Campion, A.C. Neumann, C.T. Foxon, B.L. Gallagher, and P.C. Main, Appl. Phys. Lett. 81, 3010 (2002).

${ }^{8}$ T. Jungwirth, Jairo Sinova, K.Y. Wang, K.W. Edmonds, R.P. Campion, B.L. Gallagher, C.T. Foxon, Q. Niu, and A.H. MacDonald, Appl. Phys. Lett. 83, 320 (2003).

${ }^{9}$ Jairo Sinova, T. Jungwirth, S.-R. Eric Yang, J. Kučera, and A.H. MacDonald, Phys. Rev. B 66, 041202 (2002).

${ }^{10}$ T. Dietl, H. Ohno, and F. Matsukura, Phys. Rev. B 63, 195205 (2001); M. Abolfath, T. Jungwirth, J. Brum, and A.H. MacDonald, ibid. 63, 054418 (2001).

${ }^{11}$ J. König, T. Jungwirth, and A.H. MacDonald, Phys. Rev. B 64, 184423 (2001).

${ }^{12}$ S.T.B. Goennenwein, T. Graf, T. Wassner, M.S. Brandt, M. Stutzmann, J.B. Philipp, R. Gross, M. Krieger, K. Zürn, P. Ziemann, A. Koeder, S. Frank, W. Schoch, and A. Waag, Appl. Phys. Lett. 82, 730 (2003).

${ }^{13}$ T. Shono, T. Hasegawa, T. Fukumura, F. Matsukura, and H. Ohno, Appl. Phys. Lett. 77, 1363 (2000); T. Dietl, J. König, and A.H. MacDonald, Phys. Rev. B 64, 241201 (2001).

${ }^{14}$ X. Liu, Y. Sasaki, and J.F. Furdyna, Phys. Rev. B 67, 205204
(2003).

${ }^{15}$ J.C. Slonczewski, J. Magn. Magn. Mater. 159, L1 (1996); L. Berger, Phys. Rev. B 54, 9353 (1996).

${ }^{16}$ E.B. Myers, D.C. Ralph, J.A. Katine, R.N. Louie, and R.A. Buhrman, Science 285, 867 (1999); M. Tsoi, A.G.M. Jansen, J. Bass, W.-C. Chiang, V. Tsoi, and P. Wyder, Nature (London) 409, 46 (2000); W. Weber, S. Riesen, and H.C. Siegmann, Science 291, 1015 (2001); F.J. Albert, N.C. Emley, E.B. Myers, D.C. Ralph, and R.A. Buhrman, Phys. Rev. Lett. 89, 226802 (2002).

${ }^{17}$ Ya.B. Bazaliy, B.A. Jones, and Shou-Cheng Zhang, Phys. Rev. B 57, R3213 (1998); J.C. Slonczewski, J. Magn. Magn. Mater. 195, L261 (1999); J.C. Slonczewski, cond-mat/0208207 (unpublished); M.D. Stiles and A. Zangwill, J. Appl. Phys. 91, 6812 (2002); Phys. Rev. B 66, 014407 (2002); S. Zhang, P.M. Levy, and A. Fert, Phys. Rev. Lett. 88, 236601 (2002).

${ }^{18}$ B.H. Lee, T. Jungwirth, and A.H. MacDonald, Phys. Rev. B 61, 15606 (2000); H. Ohno, D. Chiba, F. Matsukura, T. Omiya, E. Abe, T. Dietl, Y. Ohno, and K. Ohtani, Nature (London) 408, 944 (2000).

${ }^{19}$ Byounghak Lee, T. Jungwirth, and A.H. MacDonald, Phys. Rev. B 65, 193311 (2002); D. Chiba, M. Yamanouchi, F. Matsukura, and H. Ohno, Science 301, 943 (2003).

${ }^{20}$ Yaowen Liu, Zongzhi Zhang, P.P. Freitas, and J.L. Martins, Appl. Phys. Lett. 82, 2871 (2003).

${ }^{21}$ Stronger angle dependence of $\alpha$ is expected only in samples with large lattice-matching strains induced, e.g., by choosing a different III-V semiconductor as a substrate.

${ }^{22}$ S.V. Vonsovskii, Ferromagnetic Resonance (Pergamon, Oxford, 1966).

${ }^{23}$ F. Schreiber, J. Pflaum, Z. Frait, Th. Mühge, and J. Pelzl, Solid State Commun. 93, 965 (1995).

${ }^{24}$ W. Platow, A.N. Anisimov, G.L. Dunifer, M. Farle, and K. Baberschke, Phys. Rev. B 58, 5611 (1998).

${ }^{25}$ M. Tanaka and Y. Higo, Phys. Rev. Lett. 87, 026602 (2001).

${ }^{26} \mathrm{H}$. Ohno (private communication). 